\title{
Hearing loss and speech understanding in noise in Aboriginal and Torres Strait Islander children from locations varying in remoteness and socio-educational advantage
}

DOI:

10.1016/j.ijporl.2019.109741

\section{Document Version}

Accepted author manuscript

Link to publication record in Manchester Research Explorer

\section{Citation for published version (APA):}

Mealings, K., Harkus, S., Hwang, J., Fragoso, J., Chung, K., \& Dillon, H. (2020). Hearing loss and speech understanding in noise in Aboriginal and Torres Strait Islander children from locations varying in remoteness and socio-educational advantage. International journal of pediatric otorhinolaryngology, 129, 109741.

https://doi.org/10.1016/j.ijporl.2019.109741

\section{Published in:}

International journal of pediatric otorhinolaryngology

\section{Citing this paper}

Please note that where the full-text provided on Manchester Research Explorer is the Author Accepted Manuscript or Proof version this may differ from the final Published version. If citing, it is advised that you check and use the publisher's definitive version.

\section{General rights}

Copyright and moral rights for the publications made accessible in the Research Explorer are retained by the authors and/or other copyright owners and it is a condition of accessing publications that users recognise and abide by the legal requirements associated with these rights.

\section{Takedown policy}

If you believe that this document breaches copyright please refer to the University of Manchester's Takedown Procedures [http://man.ac.uk/04Y6Bo] or contact uml.scholarlycommunications@manchester.ac.uk providing relevant details, so we can investigate your claim.

\section{OPEN ACCESS}


Hearing loss and speech understanding in noise in Aboriginal and Torres Strait Islander children from locations varying in remoteness and socio-educational advantage

Kiri Mealings ${ }^{\mathrm{a}}$, Samantha Harkus ${ }^{\mathrm{b}}$, Jennifer Hwang ${ }^{\mathrm{c}}$, Jacinto Fragoso ${ }^{\mathrm{c}}$, King Chung ${ }^{\mathrm{c}}$, and Harvey Dillon a, d, e

${ }^{a}$ National Acoustic Laboratories, Sydney, Australia

${ }^{b}$ Australian Hearing, Sydney, Australia

${ }^{c}$ Northern Illinois University, United States

${ }^{d}$ University of Manchester, Manchester, United Kingdom

${ }^{e}$ Macquarie University, Sydney, Australia

\section{Corresponding Author:}

Dr Kiri Mealings

National Acoustic Laboratories

Level 5 Australian Hearing Hub

16 University Avenue

Macquarie University, NSW, 2109, Australia

Email: kiri.mealings@nal.gov.au

Funding. This study was supported by the Indigenous Advancement Strategy Project 41M7F2PW, through the Department of Prime Minister and Cabinet, Australia 
Keywords: Hearing loss; spatial processing disorder; Aboriginal and Torres Strait Islander children, conductive hearing loss, chronic middle ear disease, speech understanding in noise

Acronyms:

4FA: Four frequency average on audiometry

ANOVA: Analysis of variance

DV0: Different Voices $0^{\circ}$ Listening in Spatialized Noise - Sentences test condition DV90: Different Voices $\pm 90^{\circ}$ Listening in Spatialized Noise - Sentences test condition ICSEA: Index of Community Socio-Educational Advantage

LiSN-S: Listening in Spatialized Noise - Sentences test

SES: Socio-economic status

SPD: Spatial processing disorder

SS SiQ: Sound Scouts speech perception in quiet

SS SiN: Sound Scouts speech perception in noise

SV0: Same Voice $0^{\circ}$ Listening in Spatialized Noise - Sentences test condition

SV90: Same Voice $\pm 90^{\circ}$ Listening in Spatialized Noise - Sentences test condition 


\section{Hearing loss and speech understanding in noise in Aboriginal and Torres Strait Islander children from locations varying in remoteness and socio-educational advantage}

Objective: Otitis media resulting in conductive hearing loss is a major health issue for Aboriginal and Torres Strait Islander children, which can also lead to the child developing spatial processing disorder (SPD). This study examined the prevalence of hearing loss and deficits in speech understanding in noise, including SPD, in Aboriginal and Torres Strait Islander children from schools varying in remoteness and socio-educational advantage.

Method: 288 Aboriginal and Torres Strait Islander children aged 4-14 years from three schools varying in remoteness and socio-educational advantage completed audiological assessment and the Listening in Spatialized Noise - Sentences test to assess for hearing loss and SPD. Children also completed Sound Scouts, a selfadministered tablet-based hearing test which screens for these deficits. The prevalence of hearing issues was compared to what is expected from a typical population.

Results: The proportion of children with hearing problems was related to the school's socio-educational advantage, with higher proportions in schools with a lower socio-educational advantage. Proportions of children with speech-in-noise deficits (including SPD) was related to the remoteness of the school, with higher proportions in schools that were more remote.

Conclusions: The prevalence of hearing loss and SPD is much higher in Aboriginal and Torres Strait Islander children than described for non-Aboriginal populations, and is related to the socio-educational advantage or remoteness of the school. Resources are needed to reduce the incidence of hearing loss and health disparity in Aboriginal communities, especially those in remote areas with lower socio-educational advantages. 


\section{Introduction}

Chronic ear disease resulting in conductive hearing loss is the most prevalent health issue among Aboriginal and Torres Strait Islander children [1,2]. It is also significantly more common for this population than for non-Indigenous Australian children [3]. In all populations affected by chronic ear disease, high prevalence relates to risk factors associated with socio-economic disadvantage [4]. The World Health Organisation's report on the burden of chronic suppurative otitis media, the severest form of ear disease, positions Australian children in the category of lowest prevalence and Aboriginal Australian children in the category of highest prevalence, indicative of a 'massive public health problem requiring urgent attention' [5]. Otitis media is not just a problem for Indigenous Australians. Other countries with the highest prevalence include Tanzania, India, Solomon Islands, Guam, and Greenland [5]. A review of otitis media in Indigenous populations by Bowd [6] reports the prevalence of chronic otitis media to be 30\%-46\% in Alaskan Inuit communities, 7\%-31\% in Canadian Inuit communities, 7\%$12 \%$ in Greenland Inuit communities, $4-8 \%$ in Native American (Navajo and Apache) communities, and 8-30\% in First Nations Canadian (Cree and Ojibway) communities. The prevalence of otitis media in Indigenous children in Saskatchewan, Canada was found to be $6.7 \%$ for boys and $5.6 \%$ for girls compared to $1.1 \%$ and $0.8 \%$ for nonIndigenous children [7]. Curns et al. [8] reports that American Indian and Alaskan Indian children aged 1-4 years have otitis media-related outpatient and hospitalisation rates 1.2-1.5 times greater than non-Indigenous children. Langan, Sockalingam, Caissie, and Corsten [9] found over 20\% of First Nations children had middle ear pathology and hearing loss, and while this declined with age, children aged 6-11 years still showed abnormally high rates of middle ear pathology and hearing loss compared to nonIndigenous children. A review of otitis media in developing countries also found high 
rates of otitis media in children under six years of age, for example 10\% in Egypt, 9.2\% in Nigeria, 9.2\% in India, 9.1\% in Iran, 6.7\% in China, and 5.1-7.8\% in Russia [10]. Otitis media has also been found to be higher in children from rural compared to urban areas in America [11]. Available evidence suggests that Aboriginal \& Torres Strait Islander people began to experience significant ear and hearing health problems in the period following colonisation, as progressively members of the previously isolated population came into contact with Europeans, and thus to new, virulent strains of bacteria to which they had no immunity [12]. In remote Australia, zero of 48 children examined between 1958 and 1967 from a larger group of Aboriginal people who had no previous contact with non-Indigenous people, had evidence of chronic suppurative otitis media [13]. In contrast, between 1976-1978, 11\% of the sampled Aboriginal and Torres Strait Islander children from schools and clinics had otitis media [14].

The reduction in ear drum scarring observed in older Aboriginal adults aged over 60 years recorded during the 1976-78 National Trachoma Survey led researchers to suggest that 'Aboriginals may have more otitis media now than 40-50 years ago' [14]. This period (the 1930s) was just prior to the movement of many Aboriginal people in remote Australia from small group nomadic living to large group living in fixed and often crowded mission conditions. High rates of ear disease which continued for decades after initial exposure may be partly explained by this transition to large group and fixed abode living [12]. This factor still has relevance today as household crowding, which is a key driver of chronic ear disease, still persists in remote Aboriginal communities [15]. Crowding facilitates the spread of respiratory bacteria that cause ear disease and contribute, through over-use, to the malfunction of bathroom hardware required for controlling their spread [16,17], even in urban communities [18]. Health literacy such as the knowledge and practice of hand and face washing also plays an 
important role, so hearing loss prevention programs need to include these activities [19]. There is also evidence from epidemiologic, anatomic, physiologic, and immunologic studies that otitis media susceptibility may have a genetic component in addition to environmental and infectious factors [20].

On average, $30 \%$ of remote Aboriginal children experience chronic ear disease, but this can vary up to $90 \%$ in some communities [3]. Typically, age of onset of chronic disease is three to six months [21]. Aboriginal children in urban Australia experience similar prevalence of otitis media, but in a milder form [22]. While the prevalence of otitis media declines for non-Aboriginal children older than seven years, Aboriginal and Torres Strait Islander children can experience persistent otitis media well into their adolescence [3]. Aboriginal children are also over-represented in the Australian hearing aid-wearing child population: Australian Hearing provided audiological services, hearing aid, and cochlear implant support to 6.5\% of Aboriginal and Torres Strait Islanders compared to $0.3 \%$ of non-Indigenous children under 21 years of age [23].

Early onset otitis media per se is not now thought to pose a threat to language and communication outcomes. However, in combination with un-remediated, significant hearing loss, other health concerns, or a home environment that is not supportive of language development, otitis media is thought to present a significant risk to a child's language and communication development [24]. Poorly developed language and communication skills can in turn impact on educational engagement and outcomes, future employment options, and financial independence. Poor oral language skills are a risk factor for incarceration [25]. These problems are further exacerbated for those who are learning English as a second language [26]. 
Early onset, chronic ear disease and accompanying fluctuating hearing can also cause spatial processing disorder (SPD) in children [27,28]. Children with SPD, have abnormal difficulty understanding speech in noise because of reduced ability to take advantage of spatial separation of speech and noise to improve speech understanding in background noise despite having normal hearing thresholds [29]. Tomlin and Rance [27] found that to 6- to 12-year-old children with a history of chronic otitis media had significantly poorer listening in noise scores compared to age-matched controls. They also found that $\mathrm{z}$ scores correlated with the age of onset and duration of chronic otitis media, with poorer scores being found for children with early onset chronic otitis media and those who had it for longer periods of time.

The ability to understand speech in noise relies on the brain's skill at combining a sound's timing and level cues from both left and right auditory pathways. Fluctuating hearing in early childhood disrupts the learning of this skill. Among Aboriginal primary school children in Kempsey, New South Wales, Australia, prevalence of spatial processing disorder has previously been shown to be $7 \%$ [29]. In communities with higher prevalence of early onset, chronic ear disease it is possible that there is higher prevalence of SPD.

As socio-economic circumstances improve for Aboriginal families, fewer children are experiencing hearing loss. A large proportion of the burden of hearing loss among Aboriginal children is now mainly found among those experiencing the most socio-economic disadvantage [30], a segment of the population also less likely to have timely access to specialist otology and hearing services.

It is well established that children in Aboriginal and Torres Strait Islander communities experience conductive hearing loss more often than children in the general 
community. However, the consequences of this for speech understanding in noise in these communities is largely unknown. The purpose of this study was to examine the prevalence of hearing loss and speech understanding in noise deficits, including those arising from SPD, in Aboriginal and Torres Strait Islander communities with different proximity to major cities and different Index of Community Socio-Educational Advantage (ICSEA) which is an indication of socio-economic status. It was hypothesised that there would be a greater prevalence of hearing loss and speech in noise deficits in communities that had lower ICSEAs and/or greater remoteness.

\section{Method}

Aboriginal and Torres Strait Islander children from three schools participated in this study. They were collected in groups from their classrooms and accompanied to the testing areas which were quiet rooms in the school. Each of them completed audiometry (10 minutes), and most children completed the Listening in Spatialized Noise -

Sentences Test (LiSN-S) [31] (8-20 minutes depending on the result), and Sound Scouts (10-20 minutes depending on the version) in quiet empty classrooms or offices at the schools. Audiometry and the LiSN-S were completed individually with the examiner, whereas Sound Scouts which is a self-administered tablet-based game was completed with groups of up to four children supervised by an examiner. Children rotated through the different test stations and were given breaks between testing as required to avoid fatigue. One audiologist and three research assistants completed the testing in Campbelltown. Two audiologists and three research assistants completed the testing in Port Augusta. Three audiologists, eight audiology students, and four research assistants completed the testing in Kuranda. All examiners were trained on the tests. 


\subsection{Participants}

A total of 288 children were included in the study. There were 115 children from the school in Campbelltown, 115 children from the school in Kuranda, and 58 children from the school in Port Augusta (Table 1). Children needed to demonstrate an ability to complete the tests accurately to be included in the study. Permission to conduct this study was obtained from the Australian Hearing Human Research Ethics Committee. Parent consent was obtained, via an opt-out process, for each participating child. Figure 1 shows the distribution of ages for the participating children.

[Insert Table 1 here]

[Insert Figure 1 here]

\subsection{Audiometry}

Each child's ear canals were first examined using a Welsh Allyn Macroview otoscope. Then, the hearing sensitivity of each child was tested using a MedRX audiometer and Peltor sound-attenuating circumaural headphones integrated with D45 transducers. Conditioned play audiometry was used routinely with children aged up to six years and conventional audiometry was used for older children. In conditioned play audiometry, the child was instructed to perform a task (e.g., put a block in a bucket, put a plastic flower in a pile) whenever they heard "beeping sounds", even if the "beeps" were very faint. In conventional audiometry, the child was instructed to raise their hand when they heard a tone even if very faint.

Children's hearing thresholds were tested from $250-8000 \mathrm{~Hz}$ in octave intervals. Four frequency average (4FA) hearing thresholds were calculated using 500, 1000, 2000, and $4000 \mathrm{~Hz}$ results. If air conduction test results showed a hearing loss (thresholds of $>20 \mathrm{~dB}$ HL at any frequency), bone conduction thresholds were 
measured with masking of the contralateral ear applied as necessary. Tympanometry using an Interacoustics Titan tympanometer was also used when air conduction test results showed a hearing loss. A child was deemed to have a conductive hearing loss if they had a Type B tympanogram and/or if the child's air conduction thresholds were not within normal limits, but bone conduction thresholds were.

\subsection{Listening in Spatialized Noise - Sentences Test (LiSN-S)}

The Listening in Spatialized Noise - Sentences Test (LiSN-S) [31] measures a listener's ability to differentiate a target talker from distracting talkers by utilising interaural time and level spatial cues. The test has four conditions which are compared and used to diagnose spatial processing disorder [32-34]. In all conditions the target voice comes from the front, but the location and type of distractors differ. In the Different Voices $\pm 90^{\circ}$ condition (DV90, also known as the high-cue condition), the two different distracting voices come from $+90^{\circ}$ and $-90^{\circ}$, so the listener can use spatial and talker cues to differentiate the target voice from the distractors. In the Same Voice $\pm 90^{\circ}$ condition (SV90), the two distracting voices are spoken by the same talker as the target voice, so the listener can use only spatial cues to differentiate the target. For the Different Voices $0^{\circ}$ condition (DV0), the target and the distractors all come from the front, so the listener has to use voice cues to differentiate the target speech. Finally, in the Same Voice $0^{\circ}$ condition (SV0) the target and the distractors all come from the front and all have the same voice, so neither spatial nor talker cues can be used to differentiate the target from the distractors. This is also called the low-cue condition. The software compares these scores to calculate talker advantage, spatial advantage, and total advantage difference scores. Talker advantage is calculated as SV0-DV0. Spatial advantage is calculated as SV0-SV90. Total advantage is calculated as SV0-DV90. All 
four base scores are combined to compute an "SPD pattern measure", defined as the benefit of spatial separation, averaged across the same-voice and different-voice conditions. The software also calculates z scores (i.e. number of population standard deviations by which an individual score differs from the mean score for children of the same age) for the low-cue, high-cue, and difference scores. A listener with a spatial processing disorder would typically perform poorly on the DV90 condition and the SV90 condition (i.e. $\mathrm{z}<-2$ ), but obtain scores within normal limits on the DV0 and SV0 conditions (i.e., $z>-2$ ). This would give them a poor spatial advantage score but normal low-cue and talker advantage scores. Based on the average of (SV0-SV90) and (DV0DV90), the software combines all four scores to determine whether or not a listener has a spatial processing disorder.

The test was administered using Sennheiser HD215 circumaural headphones (Hanover, Germany) connected to a Microsoft Surface Pro 3 touchscreen computer (Microsoft, China) or iPad Air 2 (Apple Inc., California). The test is pre-calibrated so that the presentation level is always the same when it is used via the dongle for the PC version or at maximum volume on the iPad. This presentation level is $55 \mathrm{~dB}$ for the distractor tracks, and a starting level of $62 \mathrm{~dB}$ for the target sentences. The child was asked to repeat the sentences spoken by the target speaker. To minimise testing time and fatigue, testing was discontinued if a child obtained a $\mathrm{z}$ score better than -1.5 on the high-cue condition, as it is unlikely that he or she would have a spatial processing disorder with this score. Only children aged six years and above were tested on the LiSN-S as z scores are only available for children older than six years and adults $[32,33]$. Due to time constraints, not all children were tested on the LiSN-S with the numbers as follows: Campbelltown $n=114$, Kuranda $n=96$, Port Augusta $n=44$. 


\subsection{Sound Scouts}

Sound Scouts is a tablet-based hearing test designed to be completed by children older than 4 years without a clinician or clinical audiology equipment. This interactive test consists of three main measures: a speech-in-quiet calibration game; a speech-innoise game; and a tone-in-noise game. $\mathrm{Z}$ scores for children aged 4 to 18 years and adults are calculated by the app for each of these measures. In this study, we administered and analysed participants' results from the speech-in-quiet calibration game and the speech-in-noise game only. The speech-in-quiet calibration game identifies the softest speech sound that a child can understand compared to a normal hearing adult. The speech-in-noise game requires the child to identify objects spoken by a target talker while there are two competing talkers that are spatially separated from the target talker. More information on Sound Scouts can be found in Dillon, Mee, Moreno, and Seymour [35]. There are two versions of Sound Scouts - the long version which takes 20 minutes to complete (which was used in Campbelltown and Port Augusta) and a short version with reduced narration and different speech-in-quiet and tone-in-noise stimuli (but the same speech-in-noise stimuli), that takes 10 minutes to complete (which was trialled in Kuranda). The short version was designed to require a lower level of English proficiency by reducing the storyline narration, allowing for more children to be tested more quickly at school, and to be less likely to be a challenge for those children with attention difficulties. The test was administered using Sennheiser HD215 circumaural headphones (Hanover, Germany) connected to an iPad Air 2 (Apple Inc., California). The test is initially calibrated by the adult examiner with normal hearing. The first test of the game adaptively finds the speech reception threshold in quiet of the child, expressed relative to the threshold of the adult examiner. The next two tests (speech in noise and tone in noise) are presented at a specified sensation level above the 
child's speech reception threshold in quiet. This presentation level is set at $38 \mathrm{~dB}$ above the weighted average of the child's and the adult's speech reception threshold in quiet where the child:adult weighting is $80: 20$. Due to time constraints, not all children were tested on the Sound Scouts with the numbers as follows: Campbelltown $n=114$, Kuranda $n=110$, Port Augusta $n=47$.

\section{Results}

Data analysis was completed using Statistica version 13 and Microsoft Office Excel 2016.

\subsection{Otoscopy and Audiometry}

The percentage of children with middle ear problems including wax or anomalies such as a red or inflamed middle ear, perforation, retracted ear drum or dull/cloudy ear drum identified by otoscopy are shown in Table 2 . The prevalence was much higher in Port Augusta, which was both the most remote school and the school with the lowest ICSEA. Children with ear problems were referred to their local medical centre.

[Insert Table 2 here]

The children were classified into whether they had normal hearing, or a conductive or sensorineural hearing loss as determined by the audiologist using the child's pure tone audiometry air conduction thresholds, and tympanometry and bone conduction thresholds where needed to clarify the hearing loss. The results are also shown in Table 2. The differences in proportions between schools were less marked than for the observed middle ear anomalies. 
Figure 2 shows the children's 4FA hearing thresholds for left and right ears by school. The size of the bubble is related to the number of children with a particular threshold. (Note that most children were only screened down to $15 \mathrm{~dB}$ HL to avoid fatigue over the test battery.) There were various hearing profiles for the participants with a four-frequency average hearing loss for left and/or right ears of greater than 20 dB HL including flat hearing losses and reverse sloping (i.e. low frequency) hearing losses. Most children with hearing loss had a mild loss (i.e. < $40 \mathrm{~dB}$ HL). One child from Kuranda and two children from Port Augusta had moderate hearing losses in their left ears. Furthermore, one child from Campbelltown had a moderately severe bilateral loss and one child from Kuranda had a moderately severe loss in their left ear.

[Insert Figure 2 here]

\subsection{Speech Perception}

Only the results of children who had a $4 \mathrm{FA} \leq 20 \mathrm{~dB}$ HL were included in the LiSN-S analysis as even a mild hearing loss can have a negative impact on the LiSN-S results [36] (in our data, 78\% of children with hearing loss had LiSN-S high-cue condition $\mathrm{z}$ scores $<-1$ and $63 \%$ had $\mathrm{z}$ scores $<-2$, while $53 \%$ of children without hearing loss had $\mathrm{z}$ scores $<-1$ and $27 \%$ had $\mathrm{z}$ scores $<-2$ ). This gave us results for 85 children from Campbelltown, 84 children from Kuranda, and 34 children from Port Augusta. Figure 3 shows the distribution of LiSN-S high-cue condition z scores for each school. For all schools there was a skew towards poorer-than-average performance, and this skew was most profound for the school in Port Augusta. A one-way ANOVA showed this significant effect of school location on children's LiSN-S high-cue z scores $(F(2,200)=8.45, p<0.01$, mean Campbelltown $=-0.85$, mean Kuranda $=-1.27$ mean Port Augusta $=-2.29$ ). A post hoc Tukey HSD test showed significant differences 
between Campbelltown and Port Augusta $(p<0.01)$ and Kuranda and Port Augusta $(p<$ 0.05), but not Campbelltown and Kuranda $(p=0.25)$.

For the Sound Scouts, children's results were included irrespective of their audiometry performance, but only the children's test scores that the app deemed as valid (on the basis of response consistency across each adaptive test) were included in the analysis. For the school in Campbelltown, this was 109 of 114 children's speech-inquiet test scores and 106 of 114 children's speech-in-noise test scores. For the school in Kuranda, this was 94 of 110 children's speech-in-quiet test scores and 106 of 110 children's speech-in-noise test scores. For the school in Port Augusta, this was 34 of 50 children's speech-in-quiet test scores and 49 of 50 children's speech-in-noise test scores. The higher number of valid responses for the speech-in-noise test for the children from Kuranda and Port Augusta could be because this section is presented second, hence the children are more familiar with the game. All schools had similar distributions, with a skew towards poorer-than-average performance for both the speech-in-quiet and speech-in-noise measures, as shown in Figure 3. A one-way ANOVA showed no significant effect of school location on children's Sound Scouts speech-in-quiet z scores $(F(2,235)=0.04, p=0.96$, mean Campbelltown $=-0.86$, mean Kuranda $=-0.82$, mean Port Augusta $=-0.89)$, but there was a significant effect of school location on the children's speech-in-noise scores $(F(2,259)=6.42, p<0.01$, mean Campbelltown $=-0.80$, mean Kuranda $=-0.77$, mean Port Augusta $=-1.57) . \mathrm{A}$ post hoc Tukey HSD test showed significant differences between Campbelltown and Port Augusta $(p<0.01)$ and Kuranda and Port Augusta $(p<0.01)$, but not Campbelltown and Kuranda $(p=0.99)$. Fifty-two percent of children with 4FA hearing thresholds $>20 \mathrm{~dB}$ HL on pure tone audiometry had speech-in-noise z scores $<-1$, and $27 \%$ had $\mathrm{z}$ scores $<-2$. For the children without hearing loss, $43 \%$ had speech-in-noise $\mathrm{z}$ 
scores $<-1$, and $13 \%$ had $\mathrm{z}$ scores $<-2$.

[Insert Figure 3 here]

In a typical normal distribution, we would expect $12.5 \%$ of children to have $\mathrm{z}$ scores between -1 and -2 , and an additional $2.5 \%$ of children to have $\mathrm{z}$ scores $<-2$. The actual proportions falling within these ranges for the Sound Scouts speech-in-quiet (SiQ) test, the Sound Scouts speech-in-noise (SiN) test and the LiSN-S are shown in Table 3. These proportions far exceed those expected for a normal population, for all three schools. On the LiSN-S, children were considered to have SPD if the pattern measure z-score was poorer than -2, and to have a speech-in-noise deficit (caused by something other than SPD) if their z score on the high-cue condition was poorer than -2 , but they did not have SPD. The proportion with each condition is shown in Table 3 . The proportions with SPD were the same in Kuranda and Port Augusta and less in Campbelltown, but the proportion with speech in noise deficits other than SPD were much higher in Port Augusta than in the other two schools. Note that Sound Scouts only picks up children with a speech-in-noise problem, not SPD specifically, as it only has a spatially separated condition and not a collocated condition as a comparison.

[Insert Table 3 here]

\section{Discussion}

The purpose of this study was to examine the prevalence of hearing-related issues in three different Aboriginal and Torres Strait Islander communities with different proximity to major cities and different Index of Community Socio-Educational Advantage (ICSEA). Overall, it was found that the proportion of children with middle ear anomalies was greatest in the most remote school, which also had the lowest socio- 
economic status, as indexed by its ICSEA. In general, poor socioeconomic circumstances affect health throughout life [37] and social factors such as poverty, household crowding, nutrition and exposure to smoke, are known to be determinants of early onset, chronic ear disease [4]. Surprisingly, the rate of conductive hearing loss was not markedly different across the three communities.

Chronic otitis media has been linked to children developing SPD [27,28,38]. Therefore, this study also examined the prevalence of speech-in-noise deficits and SPD in children attending the three schools. The proportion of children with speech-in-noise deficits (including those for whom SPD caused or contributed to the deficit) was much higher in the most remote school, which also had the lowest ICSEA. As shown by summing columns 6 and 7 of Table 3, a total of $50 \%$ of the children tested in Port Augusta had a speech in noise deficit equivalent to two or more population standard deviations of speech understanding in noise ability on the LiSN-S. People in remote locations often have limited access to health care. Therefore, these children may have had untreated otitis media for prolonged periods of time (which seems likely given their higher rate of ear anomalies as shown in Table 2), which means they are more likely to have suffered mild-to-moderate hearing loss during this time. Having a hearing loss, whether it is permanent or fluctuating, means that the central auditory nervous system is receiving insufficient or inconsistent auditory information. This may explain why these children have developed SPD, as their auditory system has not received sufficiently clear auditory information to develop the neural pathways that process the inter-aural time and level cues needed to make use of the spatial release from masking. The prevalence of SPD in the remote and regional schools was $15 \%$. This is just over twice the prevalence found in the regional school in Cameron et al. [29], who found a prevalence of $7 \%$. While there has never been a population study to determine the 
prevalence of SPD in the general population, it is unlikely to be more than $2 \%$ based on the frequency with which outlier results were obtained during the development of normative data for the LiSN-S test [32].

Prolonged or repeated otitis media may be linked to deficits other than SPD in the developing auditory system. These include poorer dichotic perception $[39,40]$; temporal resolution [39,41]; binaural masking level differences [42,43]; very-highfrequency thresholds [44-47]. Such deficits, individually or in combination, may have contributed to the high proportion of children who had poorer than normal speech perception in noise. Speech perception in noise deficits have been directly measured in children with a history of otitis media [48-50].

It was interesting that the correlation between the LiSN-S high-cue $\mathrm{z}$ scores and Sound Scouts speech-in-noise z scores was only significant for Kuranda, and not for Campbelltown and Port Augusta. Analysis of the LiSN-S versus Sound Scouts pass/fails showed that children tended to perform more poorly on the LiSN-S, particularly in Port Augusta. This may be because the LiSN-S requires the child to repeat back sentences whereas Sound Scouts gets the child to choose from a closed set of objects, so it is easier to guess correctly. Additionally, the LiSN-S has more scored items than Sound Scouts making it a more sensitive test. It is also possible that the children found the language demands of the LiSN-S more challenging than the language demands of the speech-in-noise test of Sound Scouts, contributing to poorer scores on the LiSN-S. This is discussed further in the Limitations section.

These results show that the administration of hearing tests are needed in schools with high proportions of Aboriginal and Torres Strait Islander children, especially those in remote locations with low ICSEAs. This may be especially important for schools that have a low socio-educational advantage or are from remote locations. Not only does 
there need to be regular hearing tests, but also follow-up and treatment for those who have hearing problems. As communities that are more remote and have a lower socioeconomic status are unlikely to be able to afford to pay for regular healthcare, there is a need to make these services more accessible. Furthermore, education on hearing health and how to manage and prevent otitis media would also be beneficial for these communities. These findings are not just relevant for Indigenous Australians, but are also likely to be important for other Indigenous children around the world where otitis media is prevalent as summarised in the introduction.

\subsection{Limitations of the Study}

While this study provided insight into the prevalence of hearing-related problems in different Aboriginal and Torres Strait Islander communities, it had its limitations. First, this study only examined three schools. Therefore, we need to be cautious in drawing conclusions about the ICSEA and remoteness trends and how they relate to the prevalence of hearing or speech-in-noise deficits. The findings of this study do show some initial trends, but future studies involving a greater number of schools are needed to further explore this.

Second, both the LiSN-S and Sound Scouts tests are administered in English. For some Aboriginal and Torres Strait Islander children, as mentioned earlier, English is their second language because they grow up learning the traditional language of their community. Although the examiners administering these tests were careful in making sure the children had sufficient English to be able to complete the testing, and were more lenient when scoring the LiSN-S by allowing for grammatical errors, it is still possible that the language barrier impacted on the children's results. For some children, poor speech perception in noise could be attributed to poor understanding of the speech 
from a language perspective, rather than it being a specific speech-in-noise issue. Schafer et al. [51] found children who were native Mandarin Chinese speakers performed significantly poorer than native English speakers on the LiSN-S. Low language proficiency will increase the SRT SNR for the individual conditions of the LiSN-S, hence poor performance on the high cue condition may be due to a language issue rather than a speech-in-noise issue. However, language proficiency should have less of an effect on SPD diagnosis as it uses a difference score between the SV90 and SV0 conditions, though this is assuming that the increase in the SRT SNR as a result of the language issue is the same in both the spatially separated and collocated conditions, which it may not be. To address this language issue, we are currently developing simple language or language independent versions of these tests to avoid a person's language background influencing their test results.

Third, different versions of Sound Scouts were used at different schools. The speech-in-quiet stimuli is different in these two versions: the long version asks children to select different coloured animals, while the short version asks children to select spondee words that they have chosen from a list. Therefore there may be some differences in the results of these two tests at Kuranda versus Campbelltown and Port Augusta, though these are likely to be minimal as this section of the test is just detecting whether or not you heard the word. For the speech-in-noise test, both versions of Sound Scouts use the same stimuli and the same number of presentations, so we do not expect a difference between the two versions. The main difference between the versions is that the story narration has been reduced in the short version to make it more accessible to children with lower English proficiency.

Fourth, we were unable to take a case history with this population so we can only speculate that those children with SPD may have developed it due to prolonged 
otitis media when they were younger as found in previous studies $[27,28]$. It would be beneficial for future research to include a case history if possible so the link between those who have had chronic otitis media and those with SPD could be explored further.

\section{Conclusions}

The results of this study confirm that hearing loss and speech-in-noise deficits, particularly SPD, are much higher in Aboriginal and Torres Strait Islander children than non-Aboriginal populations, and that these conditions appear to be particularly prevalent in the more remote school with a low socio-educational advantage. The finding for the speech-in-noise deficits needs to be taken with caution, however, due to children having English as their second language which may have affected their results. Therefore, there is real need for hearing tests, including the LiSN-S or a version of it with lower demands on language proficiency, to be administered in schools with high proportions of Aboriginal and Torres Strait Islander children.

Acknowledgements. We would like to thank the participating schools and health services in the three communities including Jason Leon and Chantal Hunter from Mulungu Aboriginal Primary Health Care Service, Mark Mitchell from the Queensland Aboriginal and Islander Health Council, the audiology students from the University of Northern Illinois, Meagan Ward and Belinda Lesina from Australian Hearing, and Pam Jackson from the National Acoustic Laboratories, for their help with collecting data for this study. Harvey Dillon acknowledges the support of the Australian Department of Health, Macquarie University, and the UK National Institute for Health Research.. 
Professional meeting details. Portions of this study were presented at the Audiology Australia conference in Sydney, May 20-23, 2018, and the Otitis Media Australia conference in Darwin, August 14-16, 2018.

Funding. This study was supported by the Indigenous Advancement Strategy Project 41M7F2PW, through the Department of Prime Minister and Cabinet, Australia

\section{References}

[1] J.B. Boswell, T.G. Nienhuys, Patterns of persistent otitis media in the first year of life in aboriginal and non-aboriginal infants, Ann. Otol. Rhinol. Laryngol. 104 (1996) 542-549. doi:10.1177/000348949610501110.

[2] C. Williams, A. Jacobs, The impact of otitis media on cognitive and educational outcomes, Med. J. Aust. 191 (2009) S69-S72.

[3] Closing the Gap Clearinghouse (AIHW \& AIFS), Ear disease in Aboriginal and Torres Strait Islander children. Resource sheet no. 35., Canberra: Australian Institute of Health and Welfare, 2014.

[4] K. Kong, H.L.C. Coates, Natural history, definitions, risk factors and burden of otitis media, Med. J. Aust. 191 (2009) S39. doi:10.5694/j.13265377.2009.tb02925.x.

[5] World Health Organisation, Chronic suppurative otitis media: Burden of illness and management options, Geneva, 2004.

[6] A.D. Bowd, Otitis media: health and social consequences for aboriginal youth in Canada's north, Int. J. Circumpolar Health. 64 (2005) 5-15. doi:103402/ijch.v64i1.17949. 
[7] M. Thomson, Otitis media; how are First Nations children affected, Can. Fam. Physician. 40 (1994) 1943-1950.

[8] A.T. Curns, R.C. Holman, D.K. Shay, J.E. Cheek, L.J. Kaufman, S.F. Singleton, R.J. Anderson, Outpatient and hospital visits associated with otitis media among American Indian and Alaska native children younger than 5 years, Pediatrics. 109 (2002) e41. doi:10.1542/peds.109.3.e41.

[9] L.A. Langan, R. Sockalingam, R. Caissie, C. G., Occurrence of otitis media and hearing loss among First Nations elementary school children., Can. J. SpeechLanguage Pathol. Audiol. 31 (2007) 178-185.

[10] R. De Antonio, J. Yarzabal, J.P. Cruz, J.E. Schmidt, J. Kleijnen, Epidemiology of otitis media in children from developing countries: A systematic review., Int. J. Pediatr. Otorhinolaryngol. 85 (2016) 65-74. doi:10.1016/j.ijporl.2016.03.032.

[11] J. Bondy, S. Berman, J. Glazner, D. Lezotte, Direct expenditures related to otitis media diagnoses: extrapolations from a pediatric Medicaid cohort, Pediatrics. 105 (2000) e72. doi:10.1542/peds.105.6.e72.

[12] M. Bhutta, Evolution and otitis media: A review, and a model to explain high prevalence in indigenous populations, Hum. Biol. 87 (2015) 82-108. doi:doi: 10.13110/humanbiology.87.2.0092.

[13] J.E. Stuart, The antiquity of chronic ear disease in Australian Aboriginal children, Health History. 9 (2007) 155-158. doi:10.2307/40111580.

[14] D.J. Moran, J.E. Waterford, F. Hollows, D.L. Jones, Ear disease in rural Australia, Med. J. Aust. 2 (1979) 201-212.

[15] Commonwealth of Australia, Remote housing review: A review of the national partnership agreement on remote indigenous housing and the remote housing strategy (2008-2018), Canberra, 2018. 
[16] R. Bailie, M. Stevens, E. McDonald, D. Brewster, S. Guthridge, Exploring crosssectional associations between common childhood illness, housing and social conditions in remote Australian Aboriginal communities., BMC Public Health. 10 (2010) 147. doi:10.1186/1471-2458-10-147.

[17] P. Jacoby, K.S. Carville, G. Hall, T.V. Riley, J. Bowman, A.J. Leach, D. Lehmann, Crowding and other strong predictors of upper respiratory tract carriage of otitis media-related bacteria in Australian Aboriginal and nonAboriginal children, Pediatr. Infect. Dis. J. 30 (2011) 480-485. doi:10.1097/INF.0b013e318217dc6e.

[18] G.K.P. Spurling, D.A. Askew, P.J. Schluter, F. Simpson, N.E. Hayman, Household number associated with middle ear disease at an urban Indigenous health service: a cross-sectional study, Aust. J. Prim. Health. 20 (2014) 285-290. doi:10.1071/PY13009.

[19] E. McDonald, R. Bailie, D. Brewster, P. Morris, Are hygiene and public health interventions likely to improve outcomes for Australian Aboriginal children living in remote communities? A systematic review of the literature, BMC Public Health. 8 (2008) 153. doi:10.1186/1471-2458-8-153.

[20] M.L. Casselbrant, E.M. Mandel, The genetics of otitis media., Curr. Allergy Asthma Rep. 1 (2001) 353-357. doi:10.1007/s11882-001-0048-9.

[21] A. Leach, J. Boswell, V. Asche, T.G. Nienhuys, J.D. Mathews, Bacterial colonisation of the nasopharynx predicts very early onset and persistence of otitis media in Australian Aboriginal infants, Paediatr. Infect. Dis. J. 13 (1994).

[22] H. Gunasekera, H.M. Miller, L. Burgess, S. Chando, S.L. Sheriff, J.D. Tsembis, K.M. Kong, H.L.C. Coates, J. Curotta, K. Falster, P.B. McIntyre, E. Banks, N.J. Peter, J.C. Craig, Agreement between diagnoses of otitis media by audiologists 
and otolaryngologists in Aboriginal Australian children, Med. J. Aust. 209 (2018) 29-35. doi:10.5694/mja18.00249.

[23] Australian Hearing, Demographic details of young Australians aged less than 26 years with a hearing loss, who have been fitted with a hearing aid or cochlear implant at 31 December 2017., Sydney, 2018.

[24] J.E. Roberts, R.M. Rosenfeld, S.A. Zeisel, Otitis media and speech and language: a meta-analysis of prospective studies, Pediatrics. 113 (2004) e238-248. doi:10.1542/peds.113.3.e238.

[25] P. Snow, M. Powell, Youth (in)justice: Oral language competence in early life and risk for engagement in antisocial behaviour in adolescence, Trends Issues Crime Crim. Justice. 435 (2012) 1-6.

[26] C.J. Williams, A.M. Jacobs, The impact of otitis media on cognitive and educational outcomes, Med. J. Aust. Suppl. Otitis Media 2009 An Updat. 191 (2009) 69-72.

[27] D. Tomlin, G. Rance, Long-term hearing deficits after childhood middle ear disease, Ear Hear. 35 (2014) e233-e242. doi:10.1097/AUD.0000000000000065.

[28] K. Graydon, G. Rance, R. Dowell, B. Van Dun, Consequences of early conductive hearing loss on long-term binaural processing, Ear Hear. 38 (2017) 621-627. doi:10.1097/AUD.0000000000000431.

[29] S. Cameron, H. Dillon, H. Glyde, S. Kanthan, A. Kania, Prevalence and remediation of spatial processing disorder (SPD) in Indigenous children in regional Australia, Int. J. Audiol. (2014) 326-335. doi:10.3109/14992027.2013.871388.

[30] A. Simpson, J.C. Enticott, J. Douglas, Socioeconomic status as a factor in Indigenous and non-Indigenous children with hearing loss: analysis of national 
survey data, Aust. J. Prim. Health. 23 (2017) 202-207. doi:10.1071/PY16088.

[31] S. Cameron, H. Dillon, Listening in Spatialized Noise - Sentences test (LiSN-S) (Version 2.4), (2009).

[32] S. Cameron, H. Dillon, Development of the Listening in Spatialized NoiseSentences Test (LISN-S)., Ear Hear. 28 (2007) 196-211. doi:10.1097/AUD.0b013e318031267f.

[33] S. Cameron, H. Dillon, The listening in spatialized noise-sentences test (LISNS): Test-retest reliability study, Int. J. Audiol. 46 (2007) 145-153. doi:10.1080/14992020601164170.

[34] S. Cameron, H. Dillon, The Listening in Spatialized Noise-Sentences Test (LISN-S): Comparison to the prototype LISN and results from children with either a suspected (central) auditory processing disorder or a confirmed language disorder, J. Am. Acad. Audiol. 19 (2008) 377-391.

[35] H. Dillon, C. Mee, J.C. Moreno, J. Seymour, Hearing tests are just child's play: The Sound Scouts game for children entering school, Int. J. Audiol. 57 (2018) 529-537. doi:10.1080/14992027.2018.1463464.

[36] H. Glyde, S. Cameron, H. Dillon, L. Hickson, M. Seeto, The effects of hearing impairment and ageing on spatial processing, Ear Hear. 34 (2013) 15-28. doi:10.1097/AUD.0b013e3182617f94.

[37] R.G. Wilkinson, M.G. Marmot, Social determinants of health: the solid facts, World Health Organisation, Geneva, 2003.

[38] H. Dillon, S. Cameron, H. Glyde, W. Wilson, D. Tomlin, An opinion on the assessment of people who may have an auditory processing disorder, J. Am. Acad. Audiol. 105 (2012) 97-105. doi:10.3766/jaaa.23.2.4.

[39] L.R. Borges, J.R. Paschoal, M.F. Colella-Santos, (Central) auditory processing: 
the impact of otitis media, Clin. (Sao Paulo). 68 (2013) 954-959.

doi:10.6061/clinics/2013(07)11.

[40] L.W. Welsh, J.J. Welsh, M.P. Healy, Effect of sound deprivation on central hearing, Laryngoscope. 93 (1983) 1569-1575.

[41] B. Khavarghazalani, F. Farahani, M. Emadi, Z. Hosseni Dastgerdi, Auditory processing abilities in children with chronic otitis media with effusion, Acta Otolaryngol. 136 (2016) 456-459. doi:10.3109/00016489.2015.1129552.

[42] J.W. Hall, J.H. Grose, The effect of otitis media with effusion on the maskinglevel difference and the auditory brainstem response., J. Speech Hear. Res. 36 (1993) 210-217.

[43] D.R. Moore, M.E. Hutching, S.E. Meyer, Binaural masking level differences in children with a history of otitis media, Audiology. 30 (1991) 91-101.

[44] L.L. Hunter, R.H. Margolis, J.R. Rykken, C.T. Le, K.A. Daly, G.S. Giebink, High frequency hearing loss associated with otitis media, Ear Hear. 17 (1996) 111. https://www.ncbi.nlm.nih.gov/pubmed/8741962.

[45] P. Laitila, P. Karma, M. Sipila, M. Manninen, T. Rakho, Extended high frequency hearing and history of acute otitis media in 14-year-old children in Finland, Acta Oto-Laryngologica Supllement. 529 (1997) 27-29. https://www.ncbi.nlm.nih.gov/pubmed/9288260.

[46] R.H. Margolis, L.L. Hunter, J.R. Rykken, G.S. Giebink, Effects of otitis media on extended high-frequency hearing in children, Ann. Otol. Rhinol. Laryngol. 102 (1993) 1-5. doi:h10.1177/000348949310200101.

[47] R.H. Margolis, G.L. Saly, L.L. Hunter, High-frequency hearing loss and wideband middle ear impedance in children with otitis media histories, Ear Hear. 21 (2000) 206-211. https://www.ncbi.nlm.nih.gov/pubmed/10890728. 
[48] J.S. Gravel, I.F. Wallace, Listening and language at 4 years of age: effects of early otitis media, J. Speech Hear. Res. 35 (1992) 588-595. doi:10.1044/jshr.3503.588.

[49] A.G. Schilder, A.F. Snik, H. Straatman, P. van den Broek, The effect of otitis media with effusion at preschool age on some aspects of auditory perception at school age, Ear Hear. 15 (1994) 224-231.

[50] A. Zumach, E. Gerrits, M.N. Chenault, L.J. Anteunis, Otitis media and speech-innoise recognition in school-aged children, Audiol. Neurotol. 14 (2009). doi:10.1159/000162664.

[51] E.C. Schafer, K. Aoyama, T. Ho, P. Castillo, J. Conlin, J. Jones, S. Thompson, Speech recognition in noise in adults and children who speak English or Chinese as their first language, J. Am. Acad. Audiol. 29 (2018) 885-897. doi:10.3766/jaaa.17066. 


\section{Tables}

Table 1: Demographics of participating schools.

\begin{tabular}{llcc}
\hline Location & Remoteness & ICSEA* & $\begin{array}{c}\text { Percentage of Children } \\
\text { in Bottom SES Quarter }\end{array}$ \\
& & & 77 \\
Campbelltown, & Urban & 836 & \\
Sydney & & 46 \\
Kuranda, & Regional & 892 & \\
Northern & & \\
Queensland & & \\
Port Augusta, & Regional/Remote & 638 & \\
South & & \\
Australia & & & \\
\end{tabular}

*The Index of Community Socio-Educational Advantage (ICSEA) scale represents a school's level of educational advantage. The ICSEA scale ranges from 500 to 1300 , mean $=1000$, standard deviation $=100$. Higher ICSEA scores represent more advantaged schools. (For more information on the ICSEA scale see the My School website http://www.myschool.edu.au). We used the values calculated for 2016. 
Table 2: Percentage of children with excessive wax or otoscopically observed anomalies, and hearing loss, in each school.

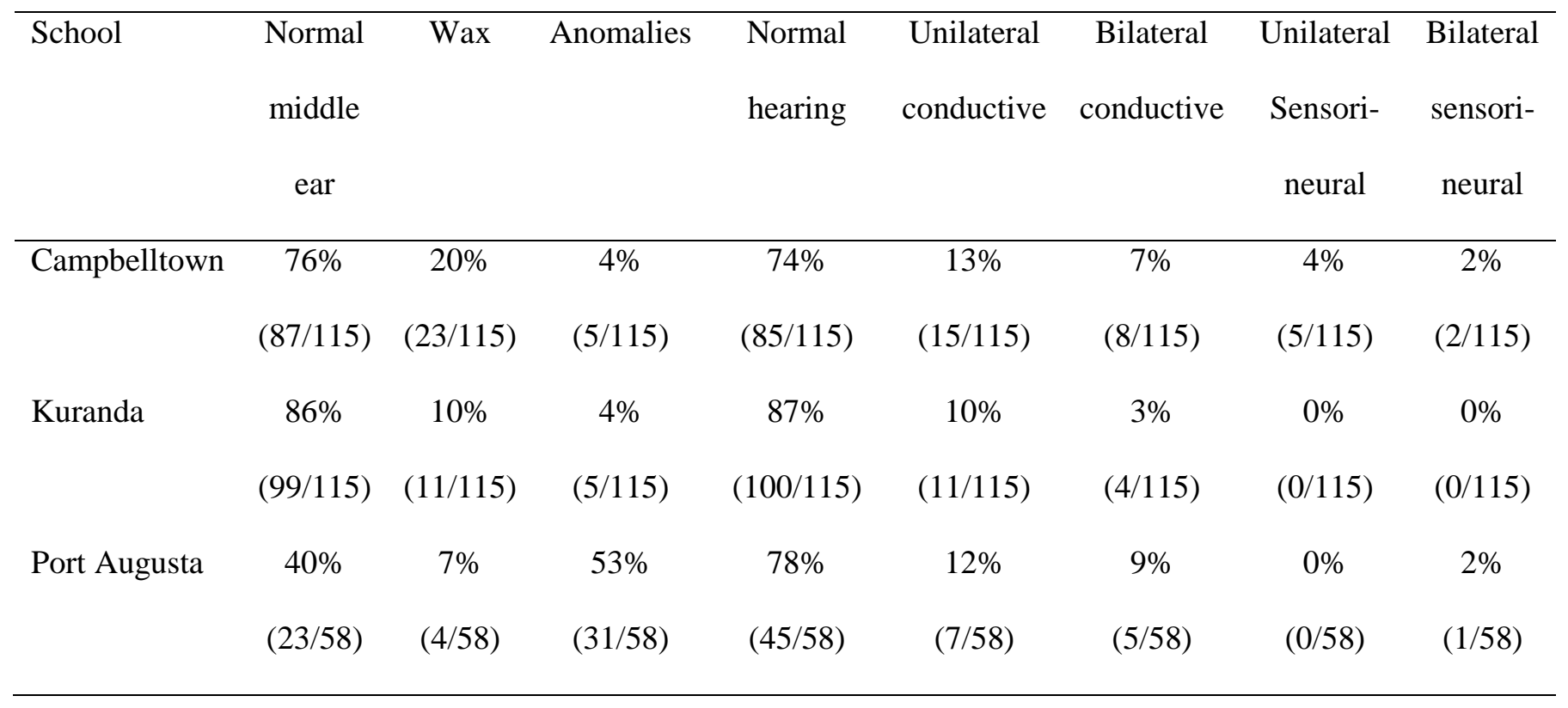


Table 3: The percentage of children with different observed speech perception deficits at each school. LiSN refers to deficits measured using LiSN-S, SS to deficits measured using Sound Scouts, $\mathrm{SiQ}$ to speech perception in quiet, and $\mathrm{SiN}$ to speech perception in noise.

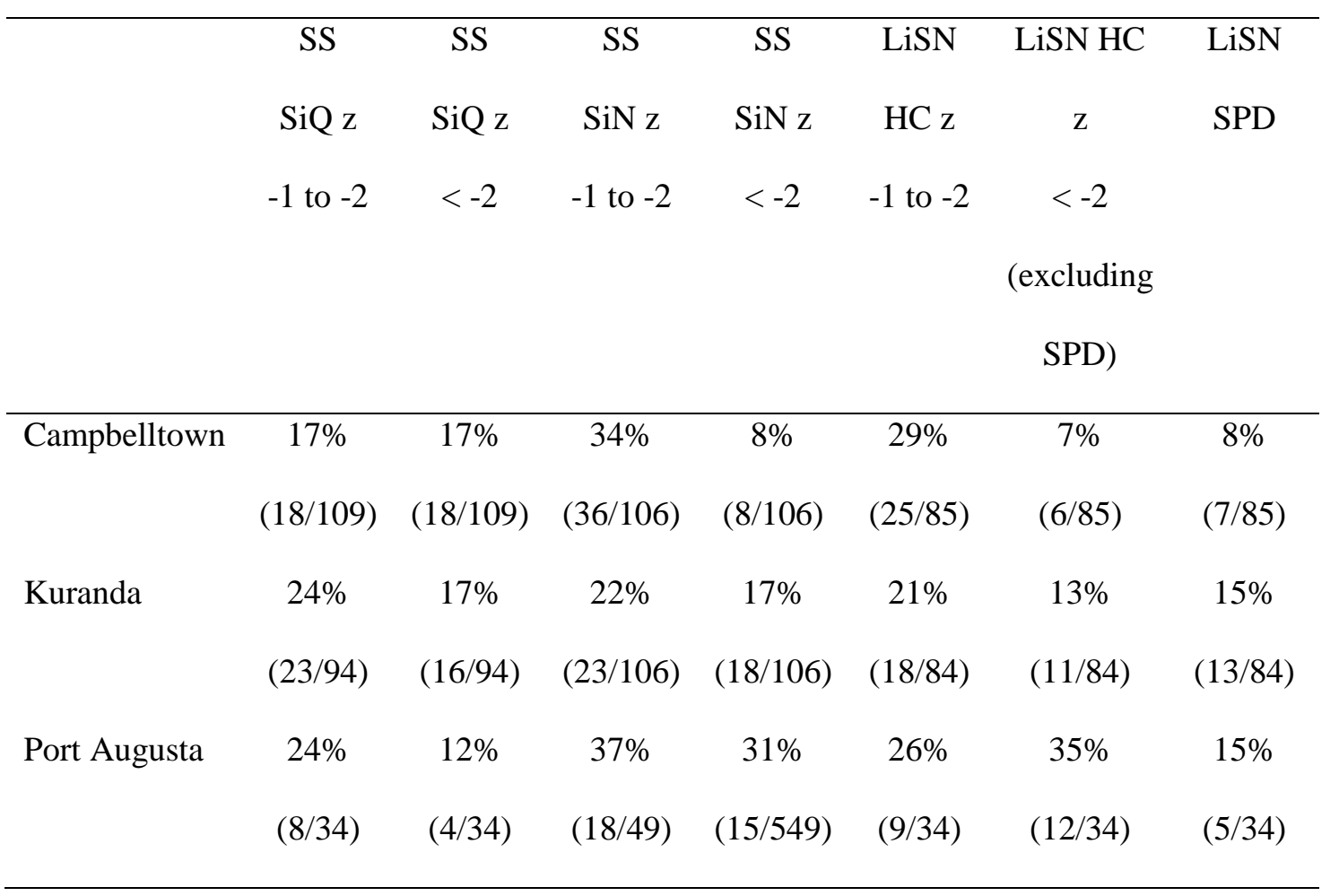




\section{Figures}

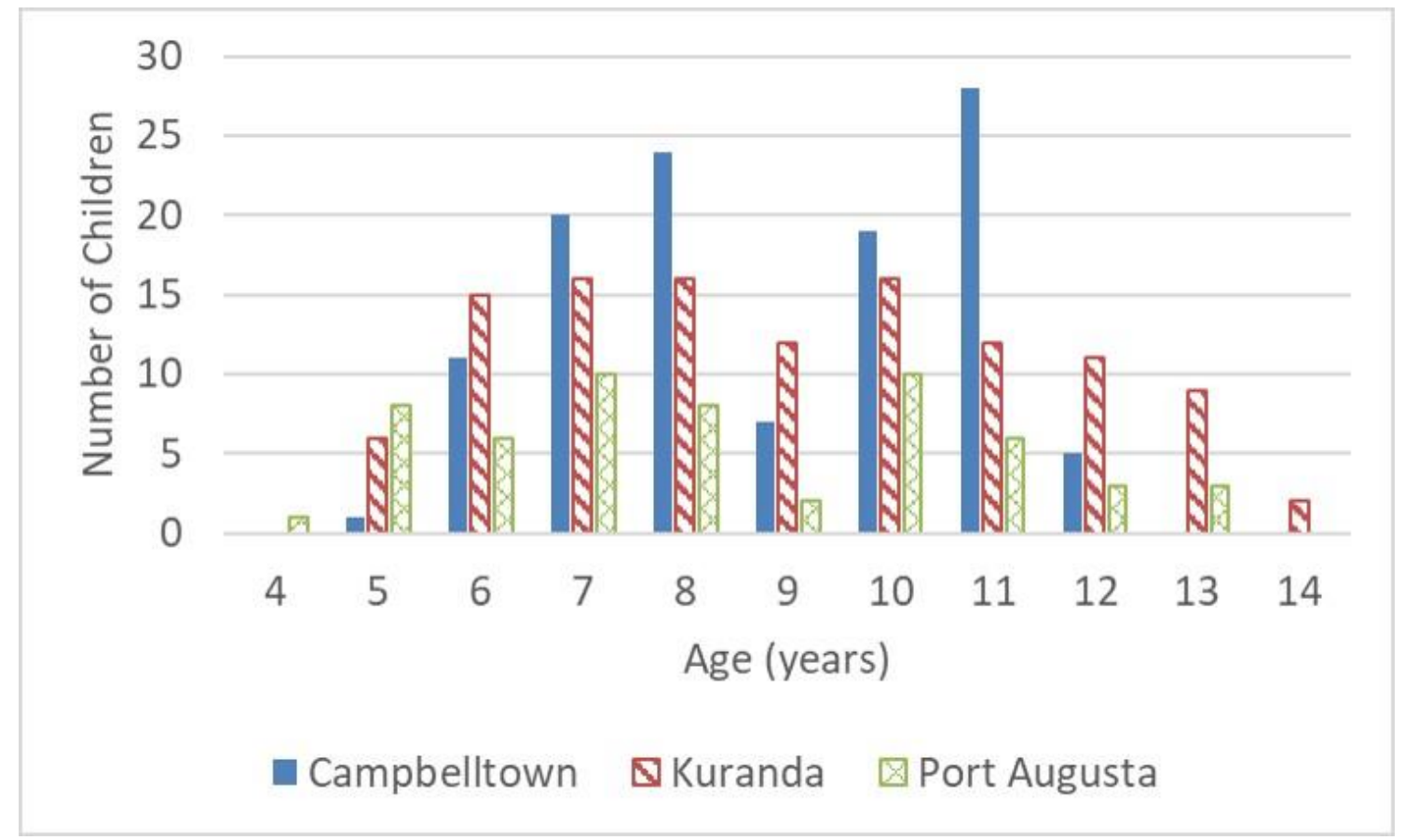

Figure 1: Distribution of ages of participating children for each school. 

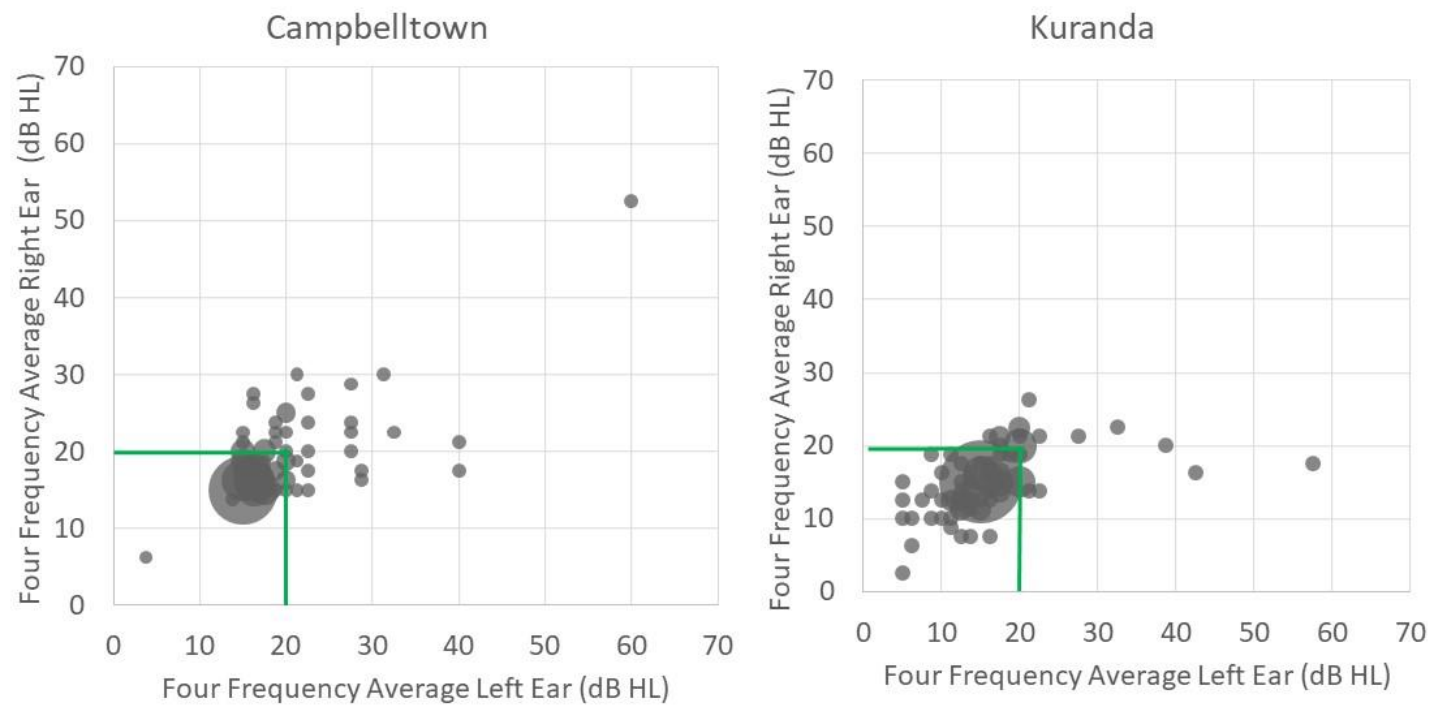

Port Augusta

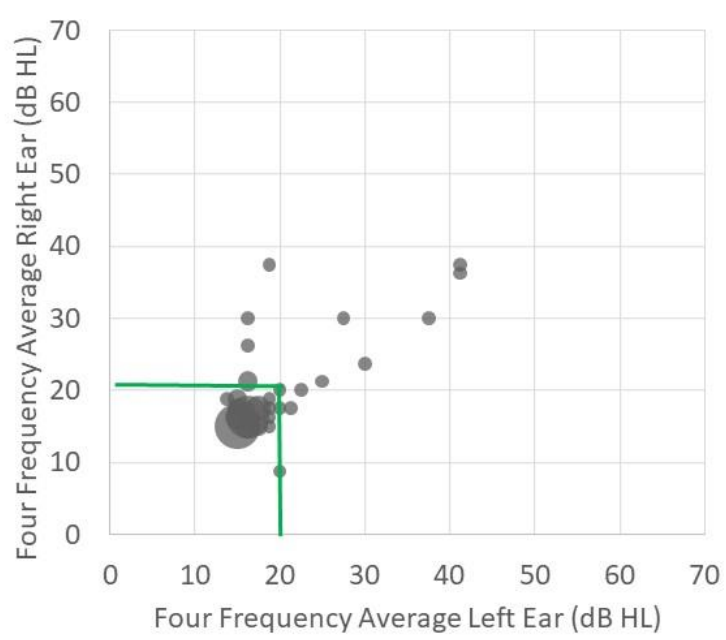

Figure 2: Participants' four frequency average hearing thresholds for left and right ears by school. Criteria for normal hearing was $4 \mathrm{FA} \leq 20 \mathrm{~dB} \mathrm{HL}$ as shown by green lines. 
LiSN-S High-Cue

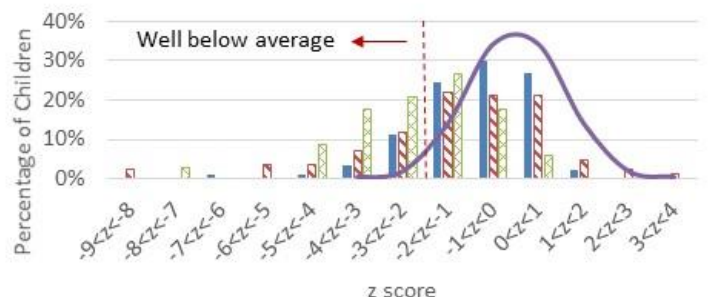

Campbelltown MWKuranda $\$$ Port Augusta — Normal

Sound Scouts Speech-in-Noise Test

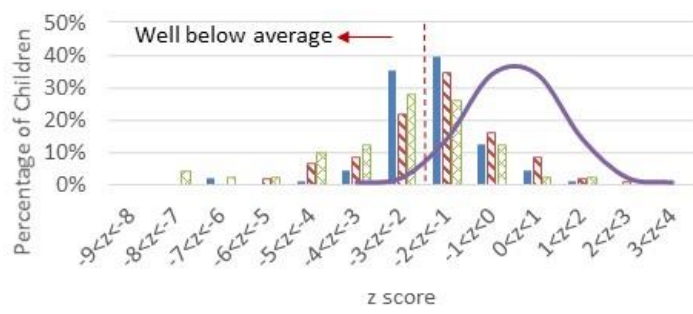

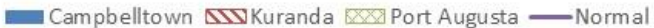

Sound Scouts Speech-in-Quiet Test

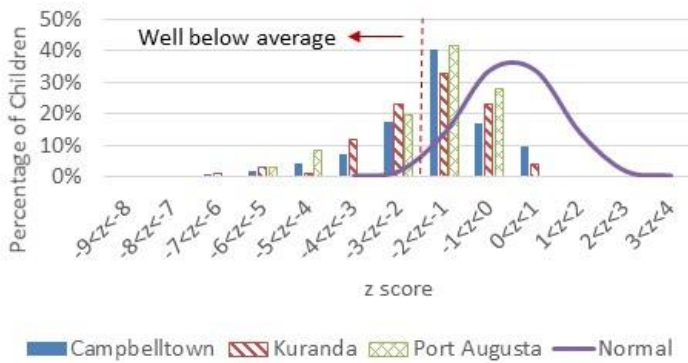

Figure 3: Distribution of z scores for each school for (a) the Sound Scouts speech-inquiet test, (b) the Sound Scouts speech-in-noise test, and (c) the LiSN-S high-cue condition compared to a normal distribution. 